\title{
Thoracic surgery and COVID-19: changes and managements during the pandemic
}

\author{
Runchen Wang ${ }^{1,2}$, Ran Zhong ${ }^{1}$, Hengrui Liang ${ }^{1}$, Tianci Zhang ${ }^{3}$, Xinkai Zhou ${ }^{4}$, Zhenyu Huo ${ }^{1,2}$, Yi Feng ${ }^{1,2}$, \\ Qixia Wang ${ }^{1,2}$, Jianfu $\mathrm{Li}^{1}$, Shan Xiong ${ }^{1}$, Feng $\mathrm{Li}^{1}$, Wenhua Liang ${ }^{1}$, Jianxing $\mathrm{He}^{1,2}$ \\ ${ }^{1}$ Department of Thoracic Surgery and Oncology, the First Affiliated Hospital of Guangzhou Medical University, State Key Laboratory of Respiratory \\ Disease, National Clinical Research Center for Respiratory Disease, Guangzhou Institute of Respiratory Health, Guangzhou, China; ${ }^{2}$ Nanshan \\ School, Guangzhou Medical University, Guangzhou, China; ${ }^{3}$ College of Cybersecurity, Sichuan University, Chengdu, China; ${ }^{4}$ School of Life \\ Sciences, Nanjing University, Nanjing, China \\ Contributions: (I) Conception and design: R Wang, R Zhong, T Zhang; (II) Administrative support: J He, W Liang; (III) Provision of study \\ materials or patients: H Liang, S Xiong, W Liang; (IV) Collection and assembly of data: H Liang, R Zhong, Q Wang, J Li; (V) Data analysis and \\ interpretation: R Wang, T Zhang, X Zhou, J Li; (VI) Manuscript writing: All authors; (VII) Final approval of manuscript: All authors. \\ Correspondence to: Jianxing He, MD, PhD, FACS, FRCS, AATS active member, ESTS member. Department of Thoracic Surgery and Oncology, \\ the First Affiliated Hospital of Guangzhou Medical University, State Key Laboratory of Respiratory Disease, National Clinical Research Center for \\ Respiratory Disease, Guangzhou, China; Southern Medical University, Guangzhou 510000, China. Email: drjianxing.he@gmail.com; Wenhua Liang, \\ MD, PhD. Department of Thoracic Surgery and Oncology, the First Affiliated Hospital of Guangzhou Medical University, State Key Laboratory of \\ Respiratory Disease, National Clinical Research Center for Respiratory Disease, Guangzhou, China. Email: liangwh1987@163.com.
}

Background: Several articles have been published about the reorganization of surgical activity during the coronavirus disease 2019 (COVID-19) pandemic but little is known about the operative volume, distribution of cases, or capacity of The Department of Thoracic Surgery to deliver surgical services in the time of COVID-19.

Methods: A retrospective operative logbook review was completed in department of thoracic in a designated COVID-19 hospital. We reviewed and analyzed the operative logbook and discussed our countermeasures during the outbreak. A prediction model was established to discuss the time consuming about delayed surgeries during the pandemic.

Results: One thousand two hundred and seventy-five operation records were collected. The thoracic surgeries of this year has decreased (43.4\%) during the Wuhan lockdown. From Jan $23^{\text {rd }}$ to Apr $8^{\text {th }}$ in 2020, there were 461 surgeries performed in The Department of Thoracic in our hospital with 0 cases of nosocomial COVID-19 infection. Prediction model showed that it will take 6 weeks to solve the backlog if department can reach the $85 \%$ of maximum of operations per week.

Conclusions: An understanding of operative case volume and distribution is essential in facilitating targeted interventions to strengthen surgical capacity in the time of COVID-19. A proper guideline is imperative to ensure access to safe, timely surgical care. By developing a scientific and effective management of hospital, it is possible to ensure optimal surgical safety during this crisis. Regular updates and a further study include multicenter is required.

Clinical trial registry number: ChiCTR2000034346.

Keywords: Coronavirus disease 2019 (COVID-19); thoracic surgery; surgical volume; surgery distribution

Submitted Sep 03, 2020. Accepted for publication Jan 07, 2021.

doi: $10.21037 /$ jtd-20-2883

View this article at: http://dx.doi.org/10.21037/jtd-20-2883

(c) Journal of Thoracic Disease. All rights reserved. 


\section{Introduction}

The coronavirus disease 2019 (COVID-19) pandemic has disrupted routine hospital services globally. Until Jul $10^{\text {th }}$, 12 million cases have been confirmed world widely with total deaths numbering over 50 thousand (1). This pandemic has had a massive impact on waits for elective operations, with tens of thousands of scheduled surgeries being cancelled or postponed (2). The post-pandemic evaluation and future planning should involve surgical services as an essential part to maintain appropriate surgical care for the population during an outbreak (3).

While there were numerous recommendations regarding surgery (4-9) and some predictive researches on the impact on surgery $(2,10)$, no researches have yet explored the surgical volumes, distribution of surgical cases, or capacity of the hospital to deliver essential surgical services in the perspective of thoracic surgery.

In China, all the COVID-19 patients were treated in designated hospitals, therefore the thoracic surgery departments in non-COVID-19 hospitals may not well develop their tailored strategies in response to the pandemic due to insufficient experience. However, all the hospitals should be cautious and be prepared for a potential second wave of the disease.

In addition, the situation on cancellation of elective surgeries is unknown and it is not clear how to address a large backlog of thoracic surgeries when surgical priorities have changed dramatically to copy with the current challenges. During the pandemic peak period in China, medical supplies especially masks and personal protective equipment (PPE) were in short supply, it could help us to perform rationally and effectively on medical resources allocation if there were a prediction model.

The aim of this article is to deliberate the above issues by conducting a retrospective study in a real-world setting based on the available data from our hospital, which is one of the four designated COVID-19 hospitals in Guangzhou. Lessons can be learned from our changes and managements in each phase of response to COVID-19. We present the following article in accordance with the MDAR reporting checklist (available at http://dx.doi.org/10.21037/jtd-20-2883).

\section{Methods}

Medical records in The Department of Thoracic Surgery were used to collect for the analysis and comparison during the Wuhan lockdown (2020, Jan $23^{\text {rd }}$ to Apr $\left.8^{\text {th }}\right)$. Hospital information system was searched to gather the surgery information. All the related information was exported in Microsoft Excel 2019 (Microsoft, Redmond, WA, USA) for inspecting and MySQL served as the relational database for continuously updated.

Two reviewers ( $\mathrm{ZR}$ and $\mathrm{RC}$ ) extracted the relevant data independently, and these data were then cross-checked. Additionally, inclusion criteria include: (I) patients who underwent surgery at the Department of Thoracic Surgery during the Wuhan lockdown; (II) patients who agreed to participate in the research and signed an informed consent form. The exclusion criteria included the following points: (I) missing detailed information; (II) patients underwent a surgery not available previously in our hospital.

We extracted the number of surgical cases, ASA grades, number of emergency/elective surgical cases and other surgical related information during the Wuhan lockdown in Department of Thoracic Surgery to compare with the paired numbers of 2019 and analyzed the distribution differences. Moreover, thoracic surgeries were classified as lung cancer surgery and non-cancer surgery according to characteristic of operation types to discuss the detailed changes. Lung cancer surgeries were classified as wedge resection, sublobar resection, lobectomy, pneumonectomy and others according to pulmonary anatomy. Non-cancer surgeries were classified as lung transplantation and others.

We here discussed our guidelines in response to this outbreak. The related control measures implemented by our hospital from the start of the outbreak were collected from the department of hospital medical affairs.

The study was conducted in accordance with the Declaration of Helsinki (as revised in 2013). The study was approved by the First Affiliated Hospital of Guangzhou Medical University (No. 2020-94) and informed consent was taken from all the patients.

\section{Statistical analysis}

A predicted model was built by using regression fitting and modeling according to data in last 5 years to research that the COVID-19 has impacted on thoracic surgery volume. Besides, we used the model to predict how long it will take in The Department of Thoracic Surgery to solve the backlog surgeries. A data analysis was performed by Python (Python version 3.7.0) on Jupyter notebook (11). Further details can be found in supplementary methods (Appendix 1). Figures were made using the drawing software Origin 2018 (OriginLab, MA, USA) and GraphPad Prism 8.0 (CA, USA). 


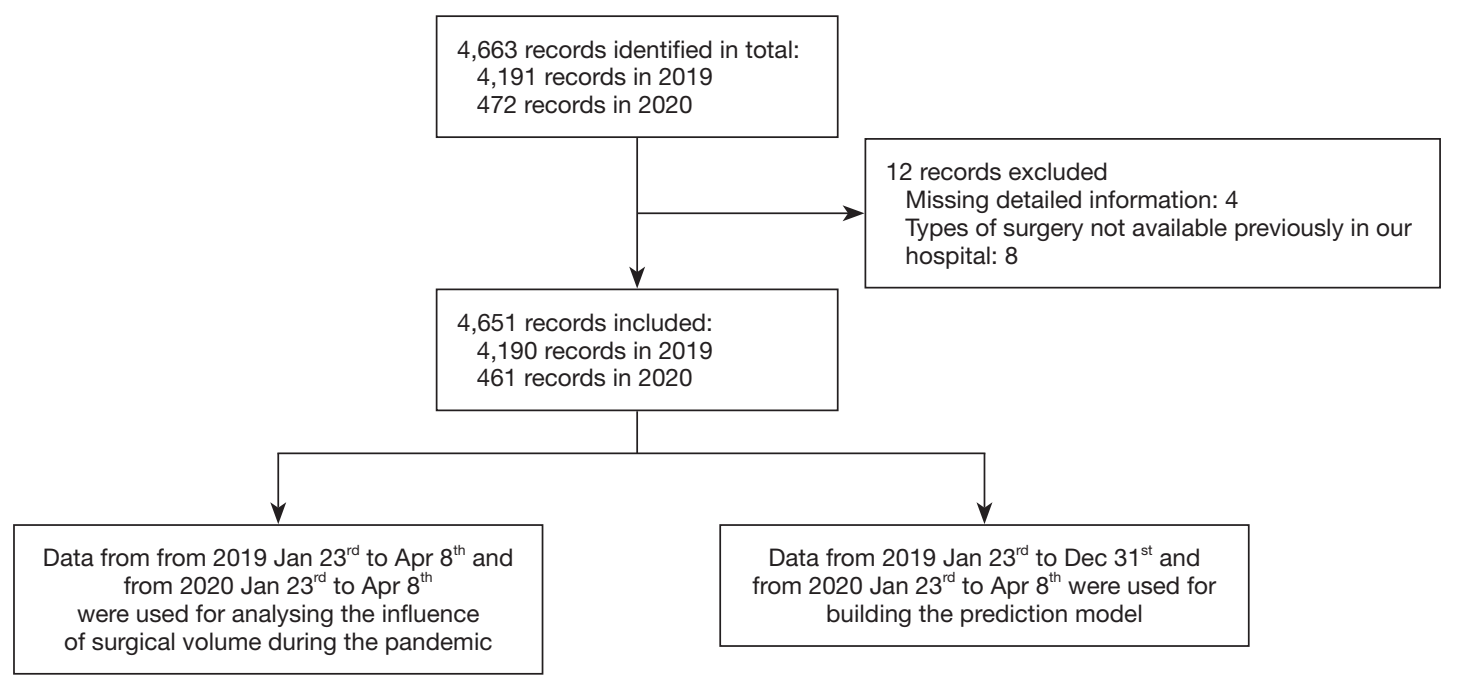

Figure 1 The flowchart of the inclusion and exclusion of the patients.

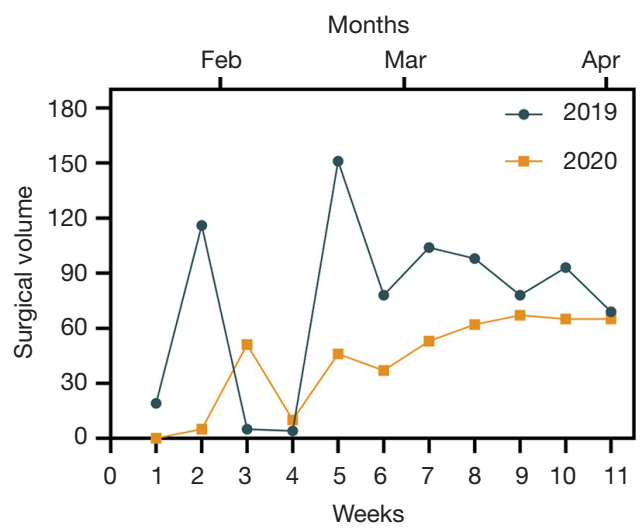

Figure 2 An overview of surgical volume during the Wuhan lockdown in our hospital.

\section{Results}

Patients from 2019 Jan $23^{\text {rd }}$ to Dec $31^{\text {st }}$ and from 2020 Jan $23^{\text {rd }}$ to Apr $8^{\text {th }}$ underwent surgery in The Department of Thoracic Surgery were reviewed consecutively. The study evaluated 4,651 (year 2019: 4,190; year 2020: 461) records for study inclusion. Among them, the data from 2019 Jan $23^{\text {rd }}$ to Apr $8^{\text {th }}$ to $2020 \mathrm{Jan} 23^{\text {rd }}$ to Apr $8^{\text {th }}$ were used to explore the impact of the thoracic surgical operation during the pandemic, and all data were used to build the prediction models. To ensure the integrity and consistency of the data for each indicator, we excluded the unqualified sample records (Figure 1).

\section{Surgical volume decreased during the COVID-19}

By comparing two sets (2019 vs. 2020) of surgical cases during the Wuhan lockdown, we found that the thoracic surgical volume in 2020 decreased overall (43.4\%) (Figure 2). To compared with the last year, a significant reduction of elective surgeries was showed (47.3\%), however, a $48.8 \%$ increase was shown in emergency surgery (Figure $3 A$, Figures S1,S2). We also compared the different types of thoracic surgeries, in all types of surgeries, the highest reduction is lung transplantation (66.7\%) (Table 1, Figure 3B). There is no obvious difference among the classification based on age, gender and anesthesia level. The changes in surgical volumes according to the ASA grades were presented in Figure S3.

\section{The outbreak response measures}

The workflow in the surgical department has changed and we believed that it will evolve as the pandemic continues. In our hospital, patients needing surgery are divided into three main categories in terms of COVID-19: infected, uncertain and uninfected. All patients were firstly arranged to a special fever clinic in our out-patient department where 2-meter distancing is required in the queue. After a temperature test, a detailed history questionnaire (especially fever and cough in the last 14 days) and physical examinations were performed by doctors with PPE.

Surgical indications were rigorously evaluated. Elective 


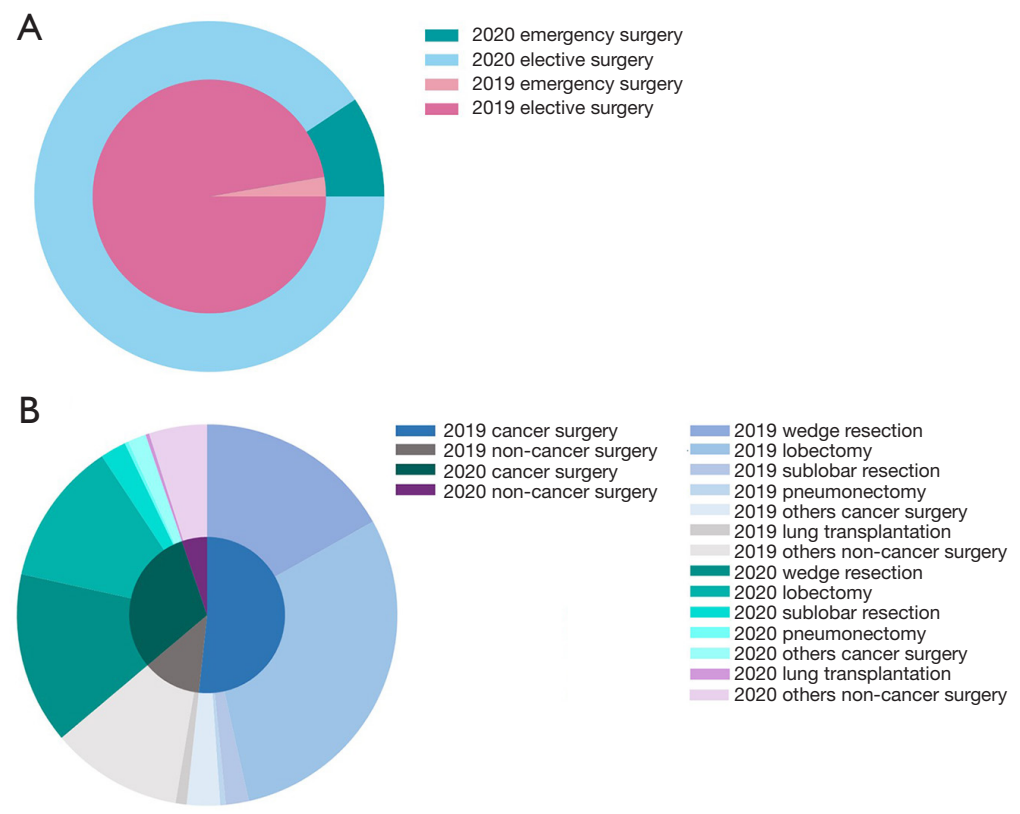

Figure 3 Comparison between 2019 and 2020 under the different operative procedures. (A) The surgical case distribution in 2 years; (B) the distribution of different types of thoracic surgeries in 2 years.

Table 1 The comparison of different types of thoracic surgeries

\begin{tabular}{lcc}
\hline Surgery type & 2019 & 2020 \\
\hline Cancer surgery & 660 & 394 \\
Wedge resection & 215 & 186 \\
Lobectomy & 378 & 156 \\
Sublobar resection & 25 & 28 \\
Pneumonectomy & 6 & 4 \\
Others & 36 & 20 \\
Non-cancer surgery & 155 & 67 \\
Lung transplantation & 12 & 4 \\
Others & 143 & 63 \\
\hline
\end{tabular}

operations for patients with stable conditions were postponed but documented and followed up through phone call. A pulmonary computed tomography (CT) scan, nucleic acid sequencing of throat swab and a complete blood count (CBC) were mandatory before hospitalization for preliminary diagnosis of COVID-19 infection.

These testing requirements were the same for any companion requesting to enter the ward as well. A patient can only have one visitor and both the patient and visitor are not allowed to leave the ward without permission during hospitalization to avoid cross infection. The mandatory COVID screening tests create additional fees (600-700 RMB) for each hospitalized person. Considering the epidemic curve is flat in China now, with no upsurge in new infections and the community spread is minimal or controlled, a tomography (CT) scan and a CBC in the above screening tests have become optional since May $20^{\text {th }}$ in our hospital, but the nucleic acid sequencing of throat swab is still a must.

Patients with positive results of COVID-19 screening tests were nursed in wards in a separate building. Patients with negative results, history of contact with confirmed cases or travel history of Wuhan were considered to be suspected cases and transferred to another specific ward. When patients were sent to hospital under a life-threating, non-COVID-19 related situation such as a car accident, emergent surgery should be performed as priority rather than the screening. Uninfected patients were transferred to the ward through a special lane to avoid cross infection. Individual accommodation was recommended for all patients and rigorous quarantine was applied for confirmed cases. Surgical management during the COVID-19 showed in Figure 4. 


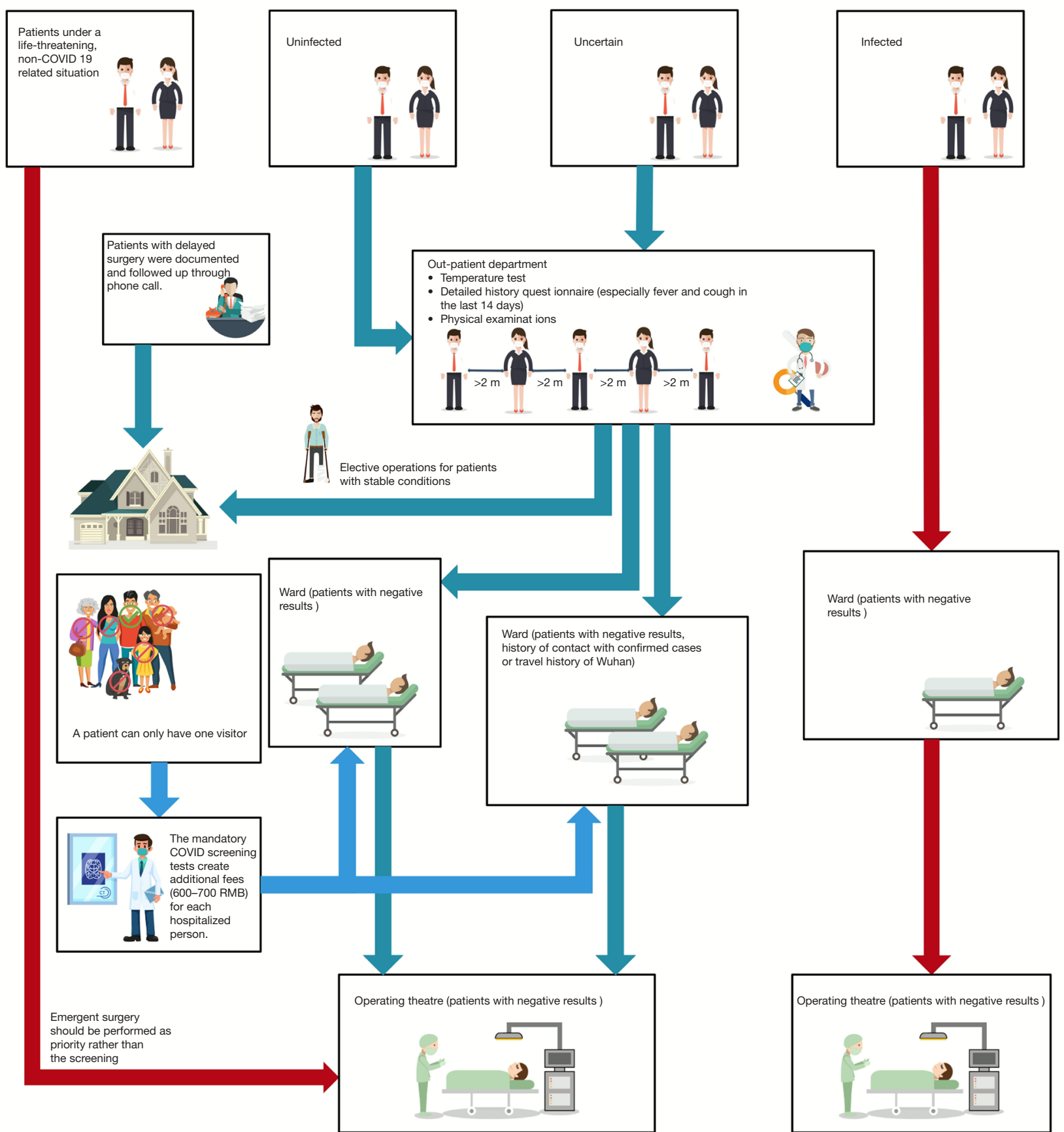

Figure 4 Surgical management during the COVID-19. COVID-19, coronavirus disease 2019.

\section{Existing data-based prediction model for delayed surgeries}

In order to predict how many manual weeks the hospital can clear the backlog surgeries, a prediction model was designed:

$$
\text { result }=129 \times p-(w \times x+b)
$$

In this equation, 129 is the max value that the hospital can operate thoracic surgery in a week, $p$ is a coefficient, $p=1$ means the all the thoracic surgeons are in position. We assume that in every week the $p$ is same. $w$ is the slope and 
the $b$ is the intercept of the regression fitting function. $x$ is the manual week (started at 12 because from Jan $23^{\text {th }}$ to Apr $8^{\text {th }}$ it passed 11 manual weeks). And by our calculates when the $p$ is smaller than 0.7856 it will appear the situation that result is negative number which means in this week the hospital, instead of operating the overstock thoracic surgery in the time period which start at Jan $23^{\text {th }}$ and end at Apr $8^{\text {th }}$, add new thoracic surgery to the overstock. So, the threshold

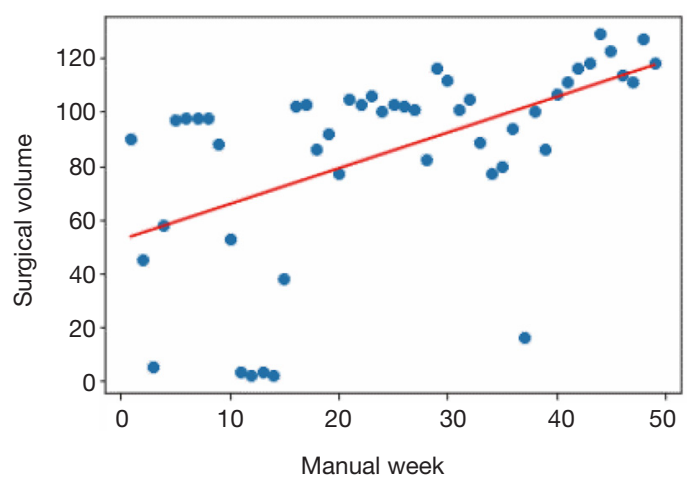

Figure 5 Fit curve of prediction model. value of $p$ is 0.7856 . Fit curve was showed in Figure 5 .

It is a remarkable fact that as for the third and fourth manual week in 2019 and the first manual week in 2020 are the time during the Chinese Spring Festival. Few surgeries are operated in these manual weeks. We regard data in these 3 manual weeks as abnormal data. In order to keep the fitting function's accuracy, we use Linear interpolation formula to simulate the thoracic surgical volume in these weeks.

Two different values of $p$-coefficient were used in this model: first, we assumed the $p$-coefficient $=0.95,0.90,0.85$, 0.80 and 0.75 which represented the all of the situation in The Department of Thoracic Surgery which exceed the threshold, time required in each situations was showed in Table 2 and Figure S4.

\section{Discussion}

The overall declined surgical volume in the Department of Thoracic Surgery during the Wuhan lockdown could be explained by multiple factors. Our hospital is one of the four designated COVID-19 hospitals in Guangzhou.

Table 2 Weekly additional surgical volume in different $p$-coefficient

\begin{tabular}{|c|c|c|c|c|c|c|c|}
\hline Weeks & \multicolumn{7}{|c|}{ Surgical volume } \\
\hline 12 & 22 & 28 & 34 & 41 & 47 & 54 & 60 \\
\hline 13 & 21 & 27 & 33 & 40 & 46 & 53 & 59 \\
\hline 14 & 20 & 25 & 32 & 38 & 45 & 51 & 58 \\
\hline 16 & 17 & 23 & 29 & 36 & 42 & - & - \\
\hline 17 & 16 & 21 & 28 & 34 & - & - & - \\
\hline 18 & 15 & 20 & 27 & - & - & - & - \\
\hline 19 & 13 & 19 & - & - & - & - & - \\
\hline 22 & 9 & - & - & - & - & - & - \\
\hline 23 & 8 & - & - & - & - & - & - \\
\hline 24 & 7 & - & - & - & - & - & - \\
\hline 25 & 5 & - & - & - & - & - & - \\
\hline 26 & 4 & - & - & - & - & - & - \\
\hline 27 & 3 & - & - & - & - & - & - \\
\hline
\end{tabular}


From Jan $23^{\text {rd }}$ to Apr $8^{\text {th }}$, there were 459 confirmed cases of COVID-19 found in Guangzhou, twenty of them were treated in our hospital. The number of blood donations has already dropped dramatically during the pandemic. The COVID-19 patients required a large amount of hospital resources such as blood plasma and many general supplies (12). Meanwhile, some of our healthcare workers were dispatched to the epicenter and the frontline healthcare workers in our hospital were rapidly altering their professional responsibilities to help hospital needs, which disarranged our normalcy. Studies have showed that in locations with widespread infections and limited resources, the risk of elective surgical procedures for patients and community may outweigh the benefit (13). As such, our hospital announced that all elective surgeries, and non-essential medical and surgical procedures be delayed at the start of the COVID-19 pandemic, which matched the guidance from The American College of Surgery posted a guideline about how to prioritize cases by describing the Elective Surgery Acuity Scale (ESAS) which has value of reference (14).

Regardless, even in the midst of a pandemic certain procedure types must be performed, including appropriate cancer treatment, emergent thoracic surgery and urgent lung transplantation, as these are considered life-saving procedures. Thus, our panelists established the rules presented in our results to ensure the safety of healthcare workers and patients involved. From Jan $23^{\text {rd }}$ to Apr $8^{\text {th }}$ in 2020 , there were 461 surgeries performed in the Department of Thoracic Surgery with zero cases of nosocomial COVID-19 infection. Despite COVID-19 being a threat, by developing a scientific and effective management of hospital, it is possible to ensure optimal surgical safety during this crisis.

Among all surgical types we reviewed in the Department of Thoracic Surgery, lung transplantation had the biggest reduction $(66.7 \%)$ on cases. Limiting access to transplantation is associated with a significant loss of prognosis for most patients on the transplant list. The influence of organ transplantation is related to the impact on the donors and increasing risks of infection.

\section{Risks of perioperative during COVID-19 pandemic}

Firstly, the lack of effective test methods during the early stage of pandemic led to the uncertainty of surgery decision. The Royal College of Surgeons recommends pre-operative chest imaging with a chest X-ray or CT, especially if the patient has respiratory symptoms and is unable to be tested for COVID-19 prior to the operating room (15). However, a large proportion of COVID-19 patients are asymptomatic. Study from University of Oxford found that the $5 \%$ and $80 \%$ of people testing positive for SARS-CoV-2 may be asymptomatic (16), which means surgeons are exposed to danger even if all subjects were negative due to the testing limitations. With the improvement of technology, Yang et al. (17) found that nasopharyngeal and oropharyngeal testing has its limitations. During the pandemic, all patients could be considered to be potentially infected with the virus given the possibility of a false negative test.

Secondly, despite the promise of accuracy of test, risks associated with intraoperation should be noted. During the operation, adequate PPE in the operating room are necessary for the health of individual healthcare providers and an adequate workforce during the pandemic. During the pandemic, minimal operators should be arranged when surgery can work correctly, which can reduce the potential scope of infection to a minimum. All the COVID-19 related surgery should be operated in negative-pressure environment. All the surgeries which can generate aerosols demanded special attention, such as thoracoscopic surgery. Furthermore, there is no evidence for assessing the impact of different types of aerosol-generating surgery on operators. Therefore, we concluded that ventilation system in operating room should be promised in a high intensity situation.

\section{Challenges in solving backlog of surgical cases}

How to decide on the optimal timing of a thoracic surgery, work within limitations and keep an eye on potential subsequent waves of COVID-19 will continue to be important.

Multiple factors are affecting the surgical plan. Firstly, hospital facilities such as shortage of operating rooms, stricter standards and more complex admission process have led a longer time to complete a surgery. Secondly, the impact of pandemic for thoracic surgery is comprehensive. Since the outbreak, blood donations in our hospital have reduced largely. Thirdly, it is hard to estimate now whether some of measures in response to COVID-19 would become "new normal" and be implemented even after this pandemic. Further investigations are needed to validate the efficacy of each measure. Surgical decision should be updated frequently, especially in a new situation $(18,19)$. 
A pulmonary CT scan, nucleic acid sequencing of throat swab and a CBC were mandatory before hospitalization for preliminary diagnosis of COVID-19 infection.

\section{Prediction model's practical application to heavily affected population}

We present proportional coefficient $p$ to represent the ability to maintain surgical volume during the COVID-19. Policymakers and hospital managers can calculate the suitable coefficient according to the severity of the pandemic and the number of surgeons locally. The proportional coefficient $p$ changes varied as the local COVID-19 pandemic situation, government measures, surgeon's completion of surgery and other factors.

The same proportional coefficient $p$ in each week was used in the design types of our research, the reason for doing so was the pandemic situation in Guangzhou and operative competence in our hospital was controllable and stable. Unless reemergence outbreak, we considered that our plan could maintain smoothly.

In the heavily affected area, millions of infected cases have been reported. The incidence rate of COVID-19 infection and the death toll is rising daily. Although we just present this notion, the coefficient can update on-the-fly to allow flexible adjustment in every week, to evaluate and master the surgical reality at the hospital. Especially true for areas with more emerging infections.

More detailed information is that in the COVID-19 designated hospitals, the ward may be occupied or isolated and procedure volume may significantly decrease. Most elective surgery can be deferred beyond the pandemic period, but emergency surgery is still necessary. These hospitals can admit patients in a planned manner according to our prediction model.

In parallel, this model can also exert its effect in the public health field, data from multiple hospitals can be collected in real-time, and the CDC can assign the hospitalization according to the reality at each hospital, which can help to solve the backlog in the whole diseaseendemic area rapidly.

\section{Advantages and shortages}

This is the first study discussed on the operative volume, distribution of cases, or capacity of a thoracic surgery departments in China to deliver surgical services in a realworld setting during this pandemic. As the escalating new
COVID-19 cases found in the world, China has entered a next phase of the COVID-19 outbreak, we hope to share our experiences and discussed the challenges we faced. Our study is also the first to build a prediction model to calculate the time required for the backlog surgeries, which can also provide a basis for next step in the multicenter research.

However, there are several shortcomings in this study. Firstly, the sample size was collected only from one hospital, which is too small to generalize the conclusion in other situations during the pandemic. Secondly, Guangzhou is a city not severely affected by COVID-19 as Wuhan in China during the period which we extracted our data from. Thirdly, compared with cities on other countries, such as America, a relatively small number of COVID-19 patients admitted in our hospital $(n=20)$ may account for a low risk of COVID-19 cross infection in our hospital, which can not be ignored when we quantify and qualify the capacity to resume surgery activity of a hospital. Fourthly, the available data only allow us to build a linear model instead of nonlinear model, which may increase the uncertainty of model.

Many factors can be found to influence the model curve, such as blood supply, which can affect the stability of our model. Meanwhile, public health emergencies can also cause the change and recalculation of predicted curve. It is pointed that regular updates based on current situation is necessary, this is why we used the MySQL served as the relational database for continuously updated. Further studies are awaited, a multicentered study on surgery and hospital managements of the whole city or country is required.

Therefore, we encourage all the health care workers around the world to share their experience and perspectives on surgery in a real-world setting during the current pandemic to collectively mitigate the impact of COVID-19.

\section{Conclusions}

An understanding of thoracic operative case volume and distribution is essential in facilitating targeted interventions to strengthen surgical capacity in the time of COVID- 19 . A proper guideline is imperative to ensure access to safe, timely surgical care. Despite COVID-19 being a threat, by developing a scientific and effective management of hospital, it is possible to ensure optimal surgical safety during this crisis.

\section{Acknowledgments}

We sincerely thank all the medical staff who are fighting 
against this public health crisis.

Funding: This work was supported by the China National Science Foundation (Grant No.81871893), Key Project of Guangzhou Scientific Research Project (Grant No. 201804020030).

\section{Footnote}

Reporting Checklist: The authors have completed the MDAR reporting checklist. Available at http://dx.doi.org/10.21037/ jtd-20-2883

Data Sharing Statement: Available at http://dx.doi. org/10.21037/jtd-20-2883

Conflicts of Interest: All authors have completed the ICMJE uniform disclosure form (available at http://dx.doi. org/10.21037/jtd-20-2883). JH serves as the Executive Editor-in-Chief of Fournal of Thoracic Disease. WL serves as an unpaid editorial board member of Fournal of Thoracic Disease from Apr 2018 to Apr 2021. The other authors have no conflicts of interest to declare.

Ethical Statement: The authors are accountable for all aspects of the work in ensuring that questions related to the accuracy or integrity of any part of the work are appropriately investigated and resolved. The study was conducted in accordance with the Declaration of Helsinki (as revised in 2013). The study was approved by the First Affiliated Hospital of Guangzhou Medical University (No. 2020-94) and informed consent was taken from all the patients.

Open Access Statement: This is an Open Access article distributed in accordance with the Creative Commons Attribution-NonCommercial-NoDerivs 4.0 International License (CC BY-NC-ND 4.0), which permits the noncommercial replication and distribution of the article with the strict proviso that no changes or edits are made and the original work is properly cited (including links to both the formal publication through the relevant DOI and the license). See: https://creativecommons.org/licenses/by-nc-nd/4.0/.

\section{References}

1. Coronavirus disease (COVID-19) pandemic. Available online: https://www.who.int/emergencies/diseases/novelcoronavirus-2019
2. Puliatti S, Eissa A, Eissa R, et al. COVID-19 and urology: a comprehensive review of the literature. BJU Int 2020;125:E7-14.

3. Søreide K, Hallet J, Matthews JB, et al. Immediate and long-term impact of the COVID-19 pandemic on delivery of surgical services. Br J Surg 2020;107:1250-61.

4. Next steps on NHS response to COVID-19: Letters from Sir Simon Stevens and Amanda Pritchard. Available online: https://www.england.nhs.uk/coronavirus/publication/nextsteps-on-nhs-response-to-covid-19-letter-from-simonstevens-and-amanda-pritchard/

5. COVID-19 and surgical procedures: a guide for patients. 2020. Available online: https://www.facs.org/covid-19/ clinical-guidance/patient-guide

6. Haddad FS. COVID-19 and orthopaedic and trauma surgery. Bone Joint J 2020;102-B:545-6.

7. Hogan A. COVID-19 and emergency surgery. Br J Surg 2020;107:e180.

8. Wexner SD, Cortés-Guiral D, Gilshtein H, et al. COVID-19: impact on colorectal surgery. Colorectal Dis 2020;22:635-40.

9. COVIDSurg Collaborative. Global guidance for surgical care during the COVID-19 pandemic. Br J Surg 2020;107:1097-103.

10. Hwee J, Chiew J, Sechachalam S. The impact of coronavirus disease 2019 (COVID-19) on the practice of hand surgery in Singapore. J Hand Surg Am 2020;45:536-41.

11. Perkel JM. Why Jupyter is data scientists' computational notebook of choice. Nature 2018;563:145-6.

12. Shander A, Goobie SM, Warner MA, et al. Essential role of patient blood management in a pandemic: a call for action. Anesth Analg 2020;131:74-85.

13. Aminian A, Safari S, Razeghian-Jahromi A, et al. COVID-19 outbreak and surgical practice: unexpected fatality in perioperative period. Ann Surg 2020;272:e27-9.

14. COVID-19: guidance for triage of non-emergent surgical procedures. 2020. Available online: https://www.facs.org/ covid-19/clinical-guidance/triage

15. COVID-19: good practice for surgeons and surgical teams. Available online: https://www.rcseng.ac.uk/standards-andresearch/standards-and-guidance/good-practice-guides/ coronavirus/

16. COVID-19: what proportion are asymptomatic? 2020. Available online: https://www.cebm.net/covid-19/covid19-what-proportion-are-asymptomatic/

17. Yang $W$, Sirajuddin A, Zhang $X$, et al. The role of imaging in 2019 novel coronavirus pneumonia (COVID-19). Eur Radiol 2020;30:4874-82. 
18. Kumar D, Manuel O, Natori Y, et al. COVID-19: a global transplant perspective on successfully navigating a pandemic. Am J Transplant 2020;20:1773-9.

19. Michaels MG, La Hoz RM, Danziger-Isakov L, et al.

Cite this article as: Wang $\mathrm{R}$, Zhong $\mathrm{R}$, Liang $\mathrm{H}$, Zhang $\mathrm{T}$, Zhou X, Huo Z, Feng Y, Wang Q, Li J, Xiong S, Li F, Liang W, He J. Thoracic surgery and COVID-19: changes and managements during the pandemic. J Thorac Dis 2021;13(3):15071516. doi: $10.21037 /$ jtd-20-2883
Coronavirus disease 2019: implications of emerging infections for transplantation. Am J Transplant 2020;20:1768-72. 


\section{Appendix 1}

We made a comparison about thoracic surgical volume in our hospital after the COVID-19 epidemic broke out with the thoracic surgery volume in last year at the same time. By using regression fitting and modeling to research the influence that COVID-19 has made on thoracic surgical volume, besides, we use the model to predict how long it will take in our hospital that recover to the thoracic surgery volume's level in last year. (Clear the volume difference in 2019 and 2020).

Data was selected from the period which start at Jan $23^{\text {th }}, 2020$, which is the date that Wuhan blockaded the city, and end at Apr $8^{\text {th }}, 2020$, which is the date that Wuhan relieved the blocking state. We counted the thoracic surgical volume in our hospital during this period, then we divided this time period by 7 days and record how many thoracic surgeries are operated in each 7 days. We regard each 7 days as a manual week so assume the volume of manual week as variable $x$, identically we assume the volume of thoracic surgery in each manual week as variable $y$. We used software method to draw the cartesian coordinate system and fit the data by Last Squares to generate a linear function and a nonlinear function, which are what we will research for.

Besides, we fitted the data in 2019 to generate functions. It is because that in the same time period in 2019 there is no COVID-19 pandemic, so we can regard the data in 2019 as the normal data. Based on this assumption, we analyzed the functions that we fitted in 2020, predicted how long it will take that recover to the last year's level after Apr. $8^{\text {th }}, 2020$.

The max weekly thoracic surgical volume was selected in 2019 as the max ability that the hospital can operate thoracic surgery. And we calculated the difference between the thoracic surgical volume during Jan $23^{\text {th }}$ to Apr $8^{\text {th }}$ in 2 years. And we discovered that the volume in 2019 is more than 2020 which means COVID-19 do influence the volume of thoracic surgery in this hospital.

\section{Research tools and environment}

We used Microsoft Excel 2019 (Microsoft, Redmond, WA, USA) as the raw data processing software, and count the thoracic surgery volume in each manual week by Pivot table function in Excel.

After we got the processing data, we saved the data as csv file, which were named as 2019_week.csv and 2020_week.csv. The content in the files are divided in two columns, the first is the number of the manual week and the second was the thoracic surgery volume in this manual week.

As for the abnormal data (the 3 manual weeks which is Chinese Spring Festival), we used Linear interpolation formula to simulate the surgery volume $(19,20)$. First, we calculate the linear function which satisfy the points on the both sides of the interpolation point, then we assume the interpolation point is on the linear function, calculate the dependent variable when the independent variable is the interpolation point. The dependent variable will be the interpolation volume to simulate.

We used Jupyter Notebook as the IDE to process the csv file under Python 3.7. In our python code, the matplotlib library is used to draw the graph and the Numpy library is used to process science calculation. Following is the core code of drawing graph:

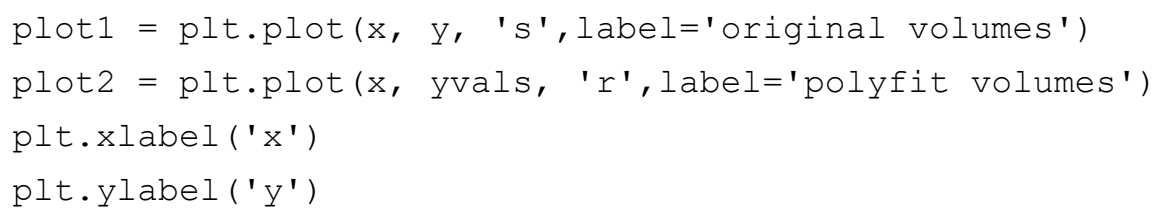




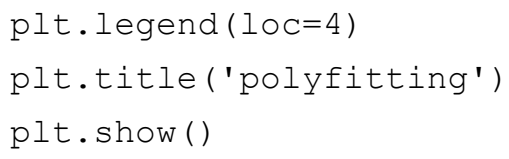

We use Last Squares method to fit the data, following is the core data of fitting:

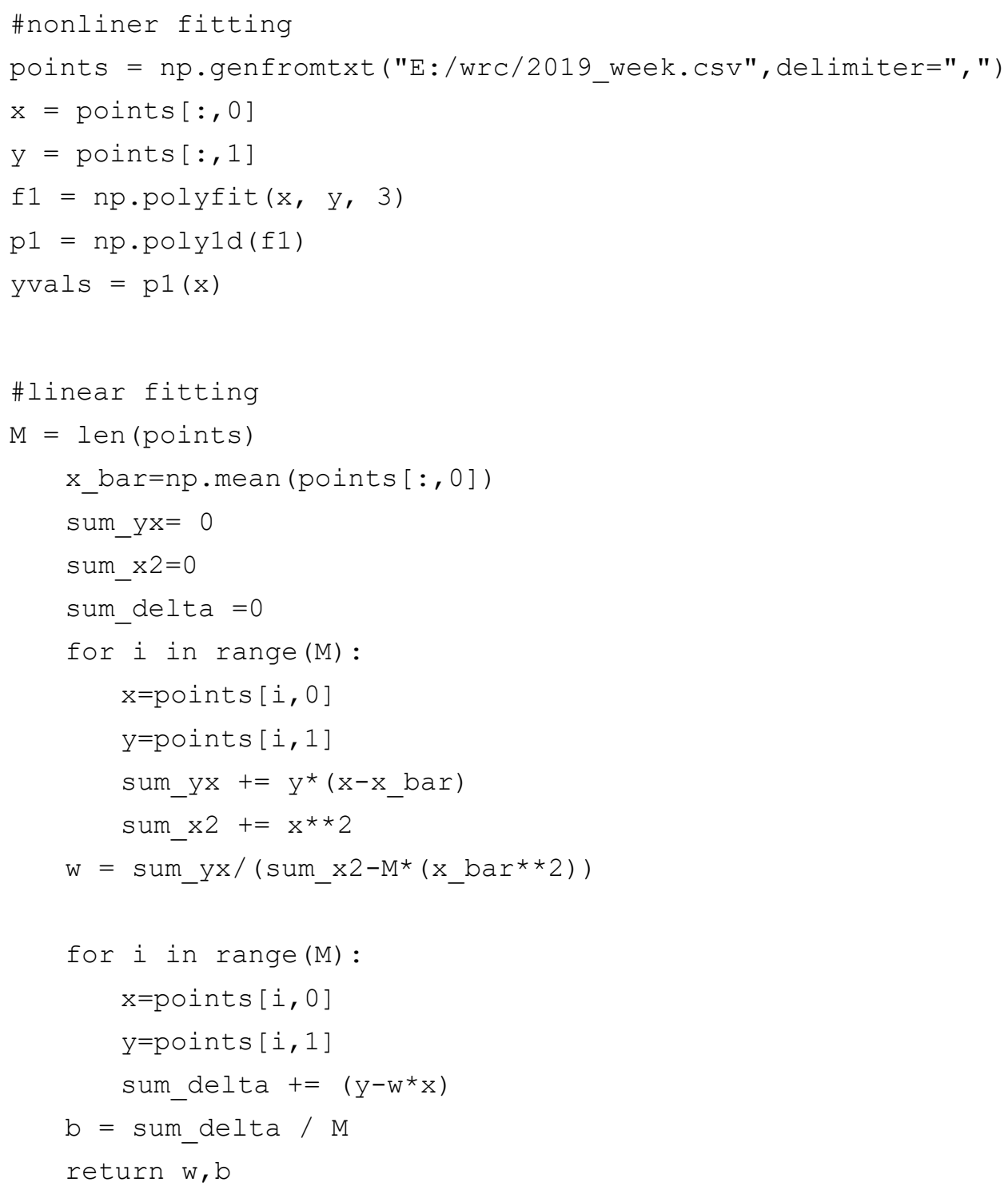

Then we use following code to calculate the difference between 2019 and 2020:

import CSV

data_2020 = Csv.reader (open("E:/wrc/final/2020_chest.csv", 'r')) 


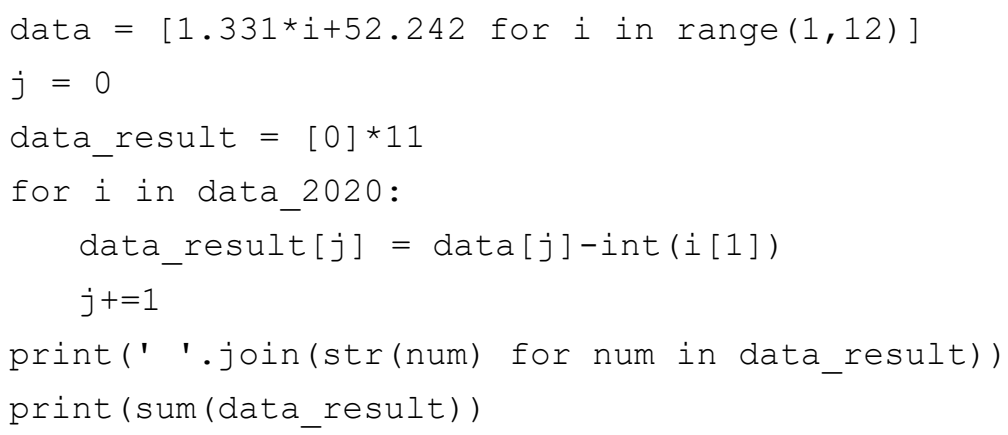

Following code is how we predict how long it will take to recover:

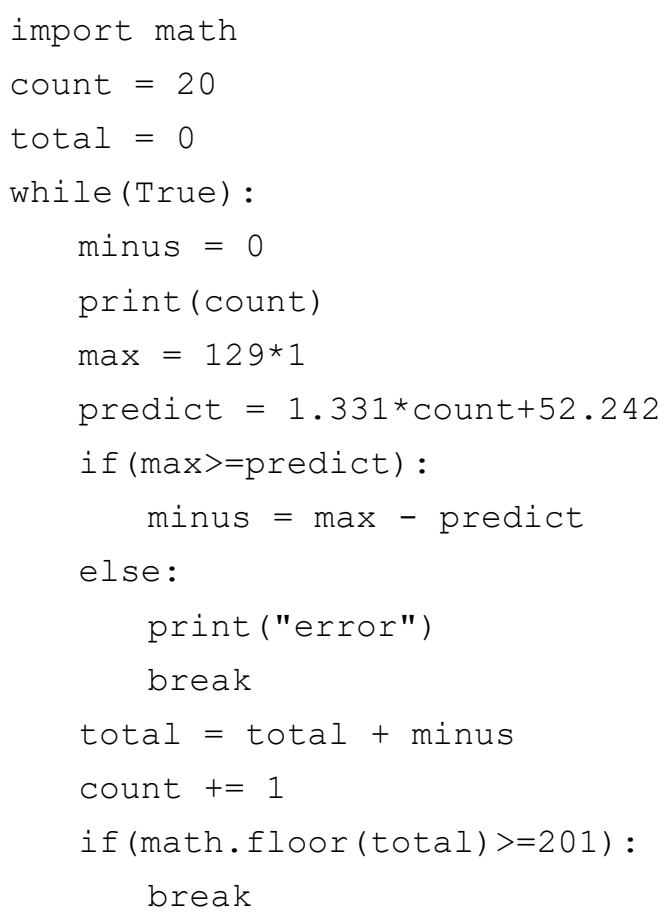

\section{References}

19. Least squares. Available online: https://en.wikipedia.org/wiki/Least_squares

20. Linear interpolation. Available online: https://en.wikipedia.org/wiki/Linear_interpolation 


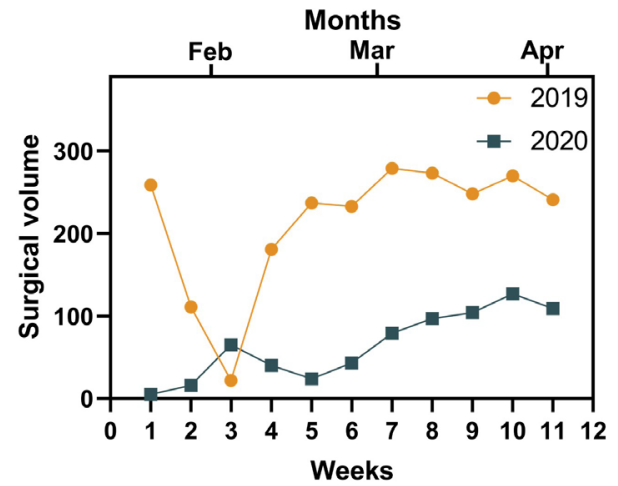

Figure S1 Changes in elective surgical volumes.

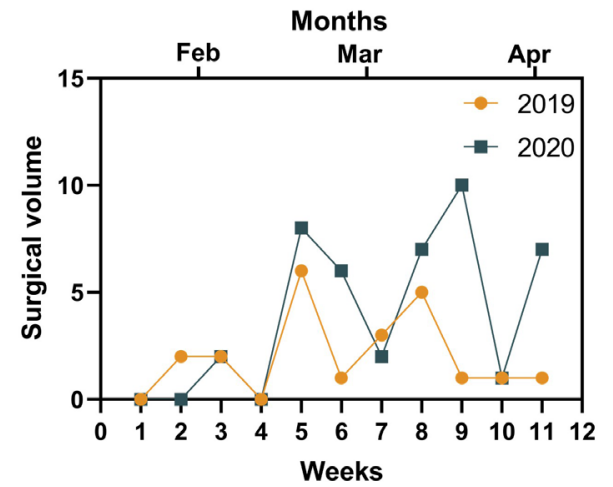

Figure S2 Changes in emergency surgical volumes.

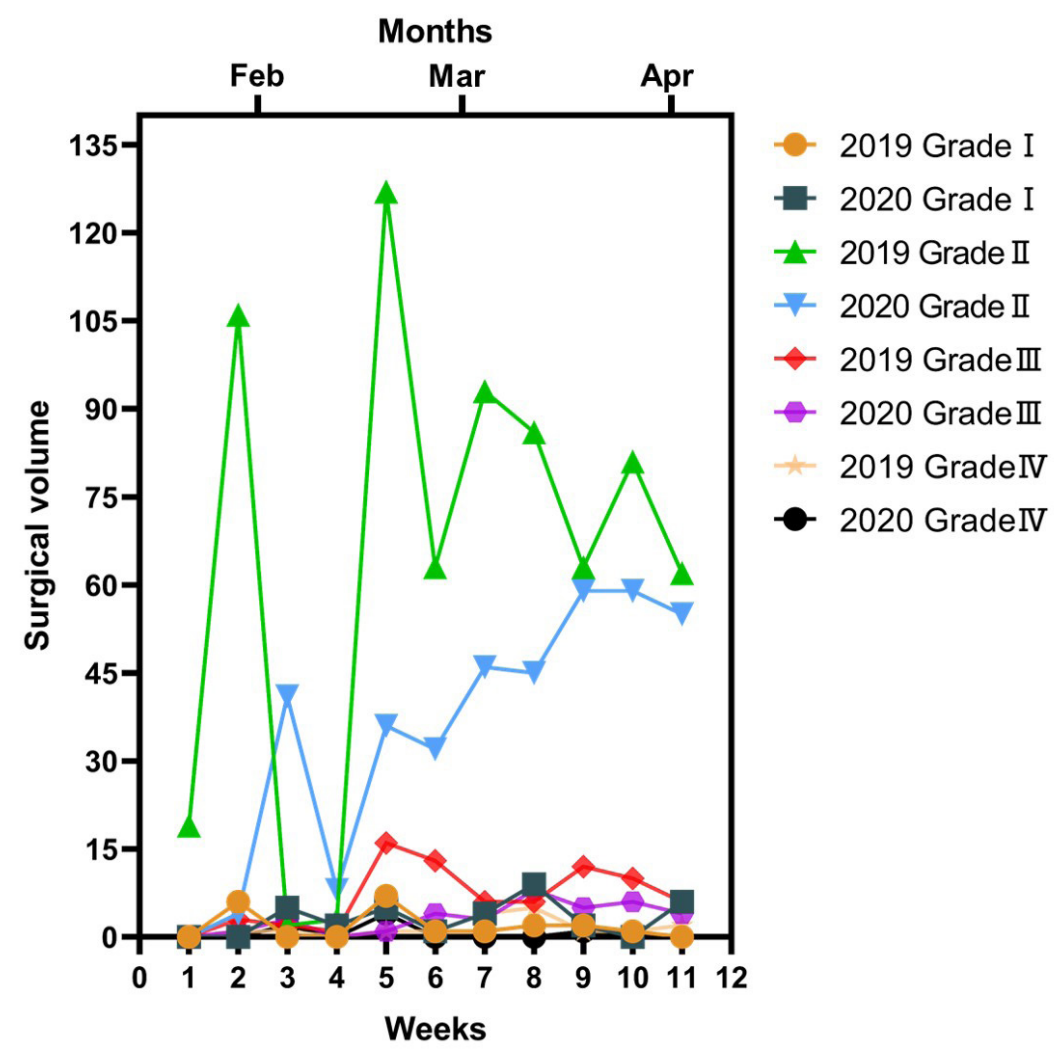

Figure S3 Changes in surgical volumes according to ASA grades. 
A
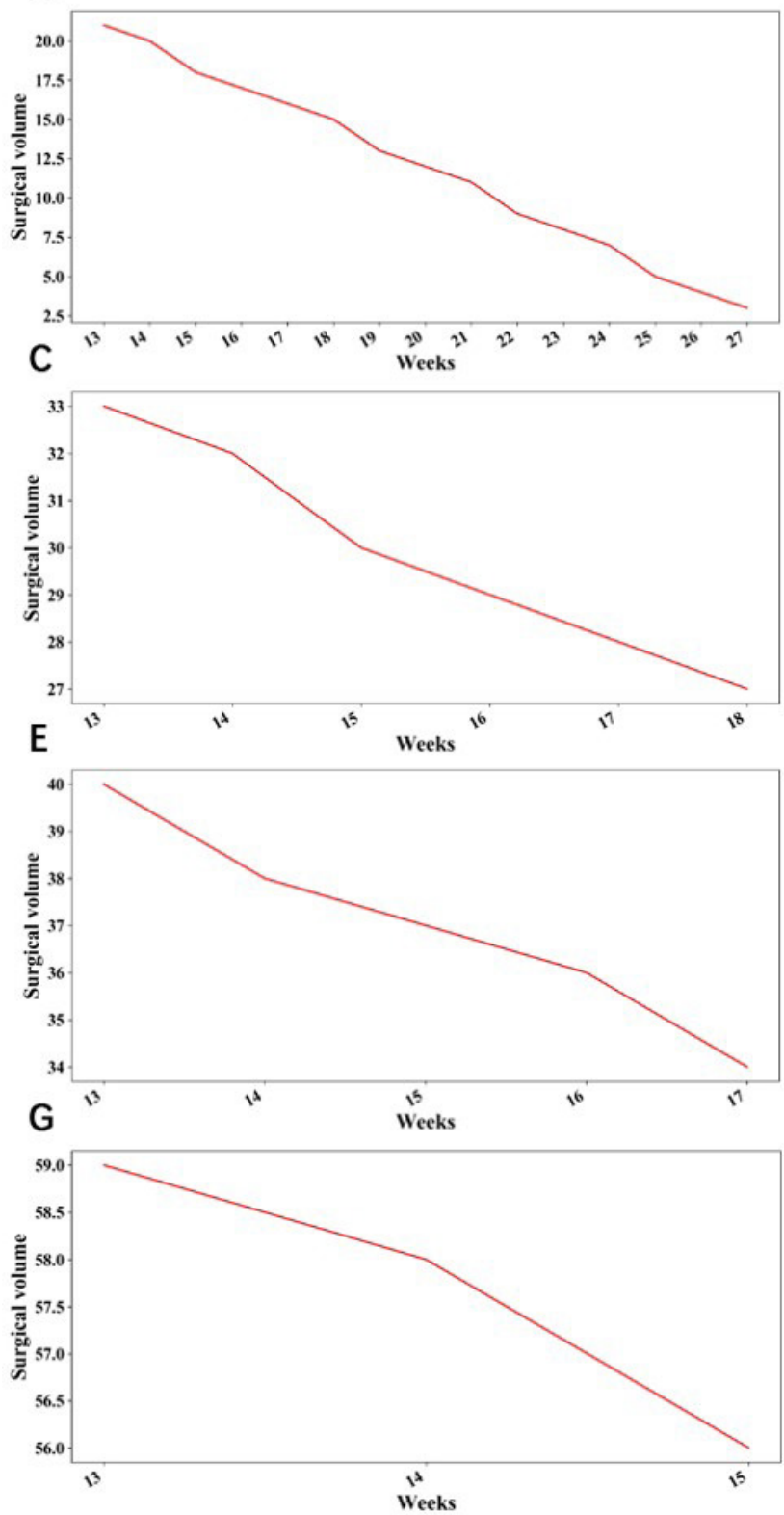

B
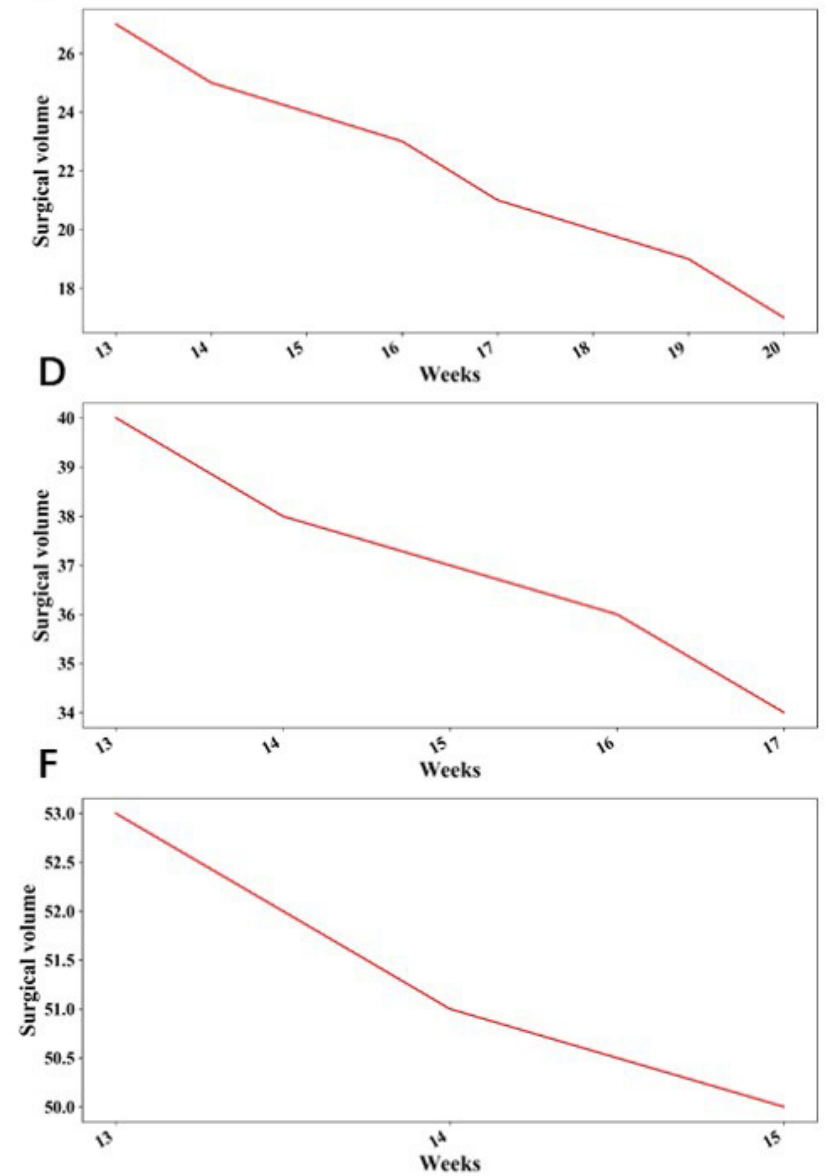

Figure S4 Time required to solve the backlog volume during the COVID-19 under the different workload in the department of surgery. (A) Time required when $p=0.70$; (B) time required when $p=0.75$; (C) time required when $p=0.80$; (D) time required when $p=0.85$; (E) time required when $p=0.90 ;(\mathrm{F})$ time required when $p=0.95 ;(\mathrm{G})$ time required when $\mathrm{p}=0.1$. *, Horizontal axis represents the number of weeks needed from the $13^{\text {th }}$ week and vertical axis represents the backlog volume. ${ }^{*}, p$ represents the proportional coefficients that the department's current surgical volume to the maximum load. COVID-19, coronavirus disease 2019. 